\title{
Quantitative Diffuse Reflectance and Diffuse Transmittance Infrared Spectroscopy of Surface-Derivatized Silica Powders
}

\author{
Farnaz Boroumand and Hubert van den Bergh \\ Laboratoire d'Etude de la Pollution Atmosphérique et des Sols, Ecole Polytechnique Fédérale de Lausanne, \\ CH-1015 Lausanne, Switzerland \\ Jacques E. Moser \\ Institut de Chimie Physique, Ecole Polytechnique Fédérale de Lausanne, CH-1015 Lausanne, Switzerland
}

\begin{abstract}
Absolute diffuse reflectance and transmittance FT-IR spectra of optically thin layers of surface-derivatized silica powders are measured, using a photopyroelectric detector placed in direct contact with the sample. The role of inhomogeneities in the surface of the powder for quantitative diffuse reflectance and transmittance measurements is examined. It is found to be equally important and nonnegligible for both sampling methods. Diffuse transmittance is shown to be more sensitive to changes of the depth of the layer than diffuse reflectance. The effect of the pressure applied to the sample surface on the scattering coefficient of two types of silica powders of different morphologies is demonstrated. A new analytical procedure based on DRIFT spectrometry is applied to the quantitative study of the influence of the chemical environment of a cyano functional group covalently linked to the silica surface on its molar extinction coefficient.
\end{abstract}

Diffuse reflectance infrared Fourier transform spectroscopy (DRIFTS) is a well-established method for qualitative analysis. Good quality spectra can be recorded using this technique without extensive sample preparation. The potential of DRIFTS for qualitative measurements was highlighted in a series of papers by Griffiths and co-workers. ${ }^{1-3}$ The capability of the method for quantitative measurements was also recognized early on. ${ }^{1,3-6}$ However, significant theoretical and practical problems arise when the method is applied for quantitative analysis. Indeed, a very precise sampling method is required in this case. Several hypotheses have to be put forward as well in order to derive various theories describing diffuse reflectance. It has often been found necessary in practice to fix experimental conditions to satisfy these hypotheses so that the appropriate models can be applied.

The effect of several parameters such as particle size, compression procedure, ${ }^{7,8}$ mechanical constraint applied on

(1) Fuller, M. P.; Griffiths, P. R. Anal. Chem. 1978, 50, 1906.

(2) Fuller, M. P.; Griffiths, P. R. Am. Lab. 1978, 10, 69.

(3) Griffiths, P. R.; Fuller, M. P. In Advances in Infrared and Raman Spectroscopy; Hester, R. E., Ed.: Heyden: London, 1982; 03

(4) Hamadeh, I. M.; King, D. J. Catal. 1984, 88, 264.

(5) Fuller, M. P.; Hamadeh, I. M.; Griffiths, P. R.; Lauenhaupt, D. E. Fuel 1982 $61,529$.

(6) Chalmers, J. M.; Mackenzie, M. W. Appl. Spectrosc. 1985, 39, 634

(7) Yeboah, S. A.; Wang, S. H.; Griffiths, P. R. Appl. Spectrosc. 1984, 38, 259.

(8) Christy, A. A.; Tvedt, J. E.; Karstang, T. V.; Velapoldi, R. A. Rev. Sci. Instrum. $1988,59,423$. the powder surface, ${ }^{9,10}$ angles of incidence and collection, ${ }^{11,12}$ and reproducibility of the preparation procedure of the sample and reference mixtures ${ }^{13}$ have been discussed in the literature.

The most ubiquitous theory describing the diffuse reflectance phenomenon was developed by Schuster ${ }^{14}$ and Kubelka and Munk. ${ }^{15,16}$ In this phenomenological model of light absorption and scattering of the powder, the sample is treated as a continuum. A simplified solution of the differential Kubelka-Munk equations is generally used in the literature to relate a chromophore concentration to the intensity of the sample's diffuse reflection:

$$
\mathcal{F}\left(R_{\infty}\right) \equiv\left(1-R_{\infty}\right)^{2} / 2 R_{\infty}=K / S \propto c
$$

In eq $1, R_{\infty}$ represents the ratio of the diffuse reflectance of the sample of interest to that of a selected reference sample. $K$ designates an absorption coefficient proportional to the concentration $c$ of the chromophore, and $S$ is the scattering coefficient of the powder. ${ }^{17}$

In such a model, the first assumption made is that the sample thickness $d$ is large enough $(d \rightarrow \infty)$ to allow the contribution of light transmission through the layer and the reflection from the background situated behind it to be neglected, i.e., $R \rightarrow R_{\infty}$. The second assumption requires that both phenomenological parameters $K$ and $S$ are taken to be constant throughout the whole sample volume. The thickness for which the diffuse transmittance is negligible depends on the absorption and scattering coefficients of the powder. In the case of an optically thin sample, i.e., if the thickness of the layer is small compared to the optical penetration depth, or if the absorption and scattering of the light inside the sample are not sufficient, part of the incident light will travel through the whole sample and be transmitted by its back surface. Normally this transmitted light is partially

(9) Murthy, R. S. S.; Leyden, D. E. Anal. Chem. 1986, 58, 1228.

(10) Chalmers, J. M.; Mackenzie, M. W. Advances in Applied Fourier Transform Infrared Spectroscopy; Wiley: Chichester, 1988.

(11) Brimmer, P. J.; Griffiths, P. R.; Harrick, N. J. Appl. Spectrosc. 1986, 40, 2581 .

(12) Brimmer, P. J.; Griffiths, P. R. Appl. Spectrosc. 1987, 4l, 791.

(13) Hamadeh, I. M.; Griffiths, P. R. Appl. Spectrosc. 1987, 41, 682

(14) Schuster, A. Astrophys. J. 1905, 21,1

(15) Kubelka, P. J. Opt. Soc. Am. 1948, 38, 448

(16) Kubelka, P.; Munk, F. Z. Tech. Phys. 1931, 12, 593.

(17) Kortüm, G. Reflectance Spectroscopy; Springer: Berlin, 1969; p 12. 
absorbed by the sample holder and partially reflected back into the sample. The real diffuse reflectance under these conditions is affected by this background reflection.

Since it is not always possible to achieve an apparent "infinite depth" using a reasonable layer thickness, in particular, in a real nondiluted system, the attempt to measure the diffuse transmittance is very interesting. This measurement provides a supplementary information channel for the determination of parameters of interest, such as the absorption and scattering coefficients. Transmittance measurements have already been performed with partially transparent selfsupporting disks using an instrument equipped with an infrared microscope. ${ }^{18,19}$

An original technique of diffuse transmittance infrared Fourier transform spectroscopy (DTIFTS) of loosely packed powders based on a novel photopyroelectric detector has been reported ${ }^{20,21}$ In a recent paper, ${ }^{22}$ general hyperbolic solutions of the Kubelka-Munk equation have been adapted to the nonideality of various analyzed systems. To do this, we have taken into account light absorption by the solid substrate, specular reflection, and the possible existence of absorption and scattering coefficients profiles within the depth of the powder layer. The adapted model was applied to relate both the intensity of diffuse reflectance and the diffuse transmittance of a silica powder to the known concentration of a cyano group covalently linked to its surface.

In the present contribution we investigate some of the experimental parameters which influence quantitative diffuse transmittance and reflectance measurements. The effect of the quality of the sample surface in both sampling methods is compared. The effect of the chemical environment on the absorption coefficient of an alkyl-cyano functional group covalently linked to a surface-derivatized silica powder is studied quantitatively.

\section{INSTRUMENTATION AND MATERIALS}

A Bomem D $A 3$ Fourier transform infrared (FT-IR) spectrometer was employed to obtain the data presented in this work, in conjunction with a short $25-\mathrm{cm}$ scan tube. The latter was especially designed for the slow mirror velocities inherent in the use of our slow pyroelectric detector that is employed in the diffuse transmittance measurements. A photopyroelectric detector assembly was used both as the sample holder for diffuse reflectance and as a detector for the diffuse transmittance infrared Fourier transform spectroscopy (DTIFTS) developed in our laboratory. The principle and details of the photopyroelectric detector have been reported elsewhere. ${ }^{23,24}$ The sample holder/detector used in this work consists of a thin (28- $\mu \mathrm{m}) \mathrm{Kynar}$ poly(vinylidene fluoride) (PVDF) film (Pennwalt Corp., Valley Forge, PA), sputtercoated on both sides with ca. $250 \mathrm{~nm}$ of Ni-Al alloy layers

(18) Fraser, D. J. J.; Griffiths, P. R. Appl. Spectrosc. 1990, 44, 193.

(19) Fraser, D. J. J.; Griffiths, P. R. Anal. Chem. 1990, 62, 308.

(20) Boroumand, F. Ph.D. Thesis, Ecole Polytechnique Fedetale de Lausanne, Lausanne, 1991.

(21) Mandelis, A.; Boroumand, F.; van den Bergh, H. Spectrochim. Acta 1991, $47 A, 943$.

(22) Boroumand, F.; Moser, J. E.; van den Bergh, H. Appl. Spectrosc. 1992, 46, 1874.

(23) Coufal, H.; Mandelis, A. Photoacoustic and Thermal Wave Phenomena in Semiconductors; North-Holland: New York, 1987; p 149.

(24) Mandelis, A.; Boroumand, F.; Solka, H.; Highfield, J.; van den Bergh, H. Appl. Spectrose. 1990, 44, 132. and inserted in an Inficon microbalance housing. ${ }^{24}$ The PVDF photopyroelectric element used for these experiments has an active area of circular cross section with a diameter of $9 \mathrm{~mm}$. To eliminate contributions from light back-reflected by the metalized PVDF surface into the powder, great care was taken to blacken that surface by depositing a layer of carbon soot on it ( $\sim 100 \mu \mathrm{m}$ thick). The sooted surface was checked to generate zero-level signals in the InSb diffuse reflectance detector. The sample holder was mechanically adapted and fitted to the "praying mantis" diffuse reflectance accessory (Harrick Scientific Corp., Ossining, NY, Model IMG 2700 L). Two wire grid KRS-5 polarizers (Harrick Scientific Corp.) were mounted in the analyzing beam of the spectrometer, one just in front of the planar entrance mirror of the accessory and the other after the exit mirror. A low noise, wide bandwidth preamplifier (Ithaco Corp., Ithaca, NY, Model 1201) was used to amplify the photopyroelectric signal to a level compatible with the A/D converter of the spectrometer $(\sim 0.1-10 \mathrm{~V})$. A high-pass first-order electronic filter was added to correct the detector response at low modulation frequencies and to eliminate low frequency noise from the interferogram that affects the reproducibility of the spectral baseline. A second amplifier compensates for the electric signal attenuation due to this filter at different frequencies. Finally, a photopyroelectric signal is delivered to the A/D converter, the amplitude of which does not vary with the modulation frequency by more than $30 \%$ between $10 \mathrm{~Hz}$ and $1 \mathrm{kHz}$. DRIFT and DTIFT spectra are obtained sequentially by switching connections from InSb detector and photopyroelectric circuit to the A/D converter, without disturbing the powder between the two measurements. In a typical experiment, a diffuse reflectance spectrum is obtained at a scan speed of $0.5 \mathrm{~cm} / \mathrm{s}$, with a resolution of $4 \mathrm{~cm}^{-1}$, by coadding 250 scans. A diffuse transmittance spectrum is typically measured at a scan speed of $0.03 \mathrm{~cm} / \mathrm{s}$, with a resolution of $4 \mathrm{~cm}^{-1}$, and by coadding 75 scans. Diffuse reflectance spectra are normalized by the spectrum of a $100 \%$ reflecting semiinfinite thickness layer of dry $\mathrm{KBr}$ powder. Diffuse transmittance spectra are normalized by the blackbody spectrum of the empty, soot-coated PVDF sample holder/ detector. In all cases, the sample compartment of the spectrometer was purged with $\mathrm{N}_{\mathbf{2}}$ to eliminate interference absorption of atmospheric $\mathrm{CO}_{2}$ and water.

Two kinds of silica powder were employed in this work. The precipitated silica LiChrosorbSi 100 (Merck, Darmstadt, Germany), characterized by a particle diameter ranging from 5 to $10 \mu \mathrm{m},{ }^{25}$ was dried at $120^{\circ} \mathrm{C}$ under vacuum before being used. The fume silica Cab-O-Sil M5 (Cabot Corp., MA) distinguishes by a primary grain diameter of $10-15 \AA$ and a secondary particle size of the agglomerated small particles of the order of $100 \AA .{ }^{26}$ This nonporous powder is submitted to consecutive thermal and hydrothermal treatments to obtain a reproducible surface for chemical derivatization..$^{20,27}$ Both silica powders are then stored and manipulated in a glovebox under a dry argon atmosphere, at a humidity level not

(25) Unger, K. K. Porous Silica; Journal of Chromatography Library Vol. 16; Elsevier: Amesterdam, 1979.

(26) Barby, D. In Characterization of Powders; Parfitt, G. D.; Sing, K. S. W., Eds.; Academic Press: London, 1976; p 389.

(27) Gobet, J.; Kováts, E. Sz. Ads. Sci. Technol. 1984, l, 77. 
exceeding $1 \mathrm{ppm}$. The specific surface area $\left(S_{\mathrm{A}}\right)$ of both silica powders was determined by evaluation of the $\mathrm{N}_{2}$ adsorption BET isotherm at $77 \mathrm{~K}$. Three independent BET measurements were performed using a Sorptomatic 1900 instrument (Carlo Erba, Milan, Italy) in a relative pressure domain ranging between 0.05 and 0.23 . Average values of $S_{\mathrm{A}}=321 \pm 3 \mathrm{~m}^{2}$ $\mathrm{g}^{-1}$ and $S_{\mathrm{A}}=191 \pm 2 \mathrm{~m}^{2} \mathrm{~g}^{-1}$ were obtained respectively for the porous LiChrosorb silica and the nonporous Cab-O-Sil.

Hydroxyl groups on the oxide surface can undergo silylation reactions with dimethylaminosilanes. LiChrosorb and CabO-Sil powders were chemically treated with two different reagents. ${ }^{20,28}$ (5-Cyano-3,3-dimethylpentyl)(dimethylamino)dimethylsilane (DMP.CN) ${ }^{29,30}$ contains a cyano functional group with a relatively sharp characteristic infrared absorption band peaking at $\mathrm{ca}$. $2247 \mathrm{~cm}^{-1}$. (3,3-Dimethylbutyl)(dimethylamino)dimethylsilane (DMB) ${ }^{29}$ has approximately the same structure as DMP.CN, but does not carry a cyano chromophore. Potassium bromide (Merck, puriss p.a. grade) powder was finely ground in a Wig-L-Bug mill capsule (Crescent Manufacturing $\mathrm{Co}$., Chicago, IL) and sieved. $\mathrm{KBr}$ particles of a diameter of $20 \mu \mathrm{m}$ or less were then dried for several hours in an oven at $180^{\circ} \mathrm{C}$ just before being used as a reflectance standard.

\section{RESULTS AND DISCUSSION}

Importance of Surface Homogeneity for Diffuse Reflectance and Transmittance Measurements. The morphology of the top surface of a sample is an important parameter for the reproducibility of diffuse reflectance measurements. ${ }^{?}$ The optical or geometrical irregularities at the surface affect the global reflectance and transmittance more than an identical perturbation in the bulk or at the bottom of the sample. To evaluate the uncertainty introduced in the quantitative measurements due to the macroscopic surface imperfections, the spectra are recorded for a sample of derivatized Cab-OSil, the surface of which is deliberately deteriorated. The powdered sample placed in the photopyroelectric detector sample holder is submitted to mechanical vibrations of about $100 \mathrm{~Hz}$ until a homogeneous 3-mm layer with a horizontal and regular looking surface is attained. The creation of small holes (ca. 10 per $\mathrm{cm}^{2}, 0.2 \mathrm{~mm}$ in diameter, and $0.2 \mathrm{~mm}$ deep) using a needle leads to a decrease of the measured reflectance by about $20-30 \%$. At the same time, diffuse transmittance intensity increases by approximately the same proportion. Such an observation shows that the two analytical channels are equally influenced by the macroscopic state of the sample surface. However, it appears that the quantitative modifications observed are much more important than is predictable for a simple local perturbation of the sample. In the absence of any compression, the thickness of the layer in these conditions remains effectively constant. The systematic decrease of reflectance and the increase of the transmittance intensity therefore suggest a more efficient penetration of light into the bulk of the sample due to a waveguide-like effect. The sensitivity of these quantitative measurements to the surface quality of the analyzed sample hence requires the use of a

(28) Erard, J. F.; Nagy, L.; Kováts, E. Sz. Colloids Surf. 1984, 9, 109.

(29) Szabo, K.; Ha, N. L.; Schneider, P.; Zeltner, P.; Kováts, E. Sz. Helv. Chim. Acta 1984, 67, 2128.

(30) Schneider, P.; Cloux, R.; Fóti, K.; Kováts, E. Sz. Synthesis 1990, 1027. procedure which ascertains the regularity and the reproducibility of the macroscopic surface of the layer. Two methods have been employed so far with various success:

The first one consists of filling the sample holder with an excess of powder, which is then eliminated by scraping the top of the cup with a spatula. The second one proposed by Yeboah et al. ${ }^{7}$ proposes the application of a vertical pressure on the powder surface. These authors showed for their system that such a procedure provides a better reproducibility compared to the spatula method, which is very much dependent on the dexterity of the operator. In the case of pressure application, the result depends essentially on the applied force, the geometry of the initial powder heap, and the achieved compression ratio.

Our experiments have been carried out with 3-mm-thick samples of various mixtures of LiChrosorb powders chemically treated by DMB and DMP.CN aminosilanes, respectively. Four batches of the powder mixture are prepared each time for each of the 10 various concentrations of DMP.CN substituents used. The spatula technique allows a reproducibility of $\pm 3 \%$ on the reflectance measurements at a wavenumber $\bar{\nu}=2247 \mathrm{~cm}^{-1}$, which corresponds to the maximum absorption of the cyano group.

To control the applied force on the powder surface, a dynamometric press has been designed in our laboratory. A polished stainless steel piston is operated vertically by a rigid lever on which different brass masses can be hung at various distances from the horizontal axis. The piston is gradually lowered on the sample, on which it can apply a determined pressure during a known period of time. In all except one series of experiments, particularly designed to study the effect of powder compression, the sample cup is filled with a constant excess of silica, and a piston of a diameter larger than that of the cup is used. Since the stroke of the piston is limited by the top of the cup, most of the excess powder is removed, and the pressure is only partially transmitted to the powder. This method minimizes the compression ratio, which is at most 1.05 for the LiChrosorb powder and 1.25 for the less dense and more compressible $\mathrm{Cab}-\mathrm{O}-\mathrm{Sil}$ powder. The reproducibility of the measurements is in this case $\pm 3 \%$, similar to that of the spatula method for the same operator. The pressure application technique is more complex, but its undisputed advantage lies in the possibility of producing optically reproducible surfaces essentially independent of the operator. However in the case of the transmittance measurements, this method cannot be employed with the present experimental configuration. Even a small pressure transmitted to the bottom of the powder is sufficient to modify the sensitive PVDF element, which is also known to display strong piezoelectric properties. This problem prevents the comparison of the sample transmittance intensity with the one corresponding to the empty blackened detector.

The homogenization of thin powdered samples by mechanical vibration is an alternative for the leveling of their surface. The variation recorded for eight independent reflectance and transmittance measurements of a $300-\mu \mathrm{m}$ thick layer of a mixture of treated Cab-O-Sil powders is about $6 \%$ in both cases. The reproducibility for this preparation method thus appears to be somewhat poorer than that obtained by the pressure application technique used for thicker samples. 
Table 1. Normallzed Diffuse Reflectance and Tranemittance of $K B$ Powder Layers of Varlous Thleknesces on

\begin{tabular}{llll}
\multicolumn{1}{c}{$d(\mathrm{~cm})$} & \multicolumn{1}{c}{$R / R_{\infty}$} & \multicolumn{1}{c}{$T / T_{0}$} & $R / R_{\infty}+T / T_{0}$ \\
0 & 0 & 1 & 1 \\
$0<d_{1}<0.3$ & $0.55 \pm 0.03$ & $0.42 \pm 0.02$ & $0.97 \pm 0.05$ \\
$0<d_{2}<0.3$ & $0.90 \pm 0.05$ & $0.13 \pm 0.01$ & $1.03 \pm 0.06$ \\
0.3 & 1 & 0 & 1
\end{tabular}

a The uncertainties on diffuse reflectance and transmittance measurements are estimated at about \pm 6 and $\pm 5 \%$, respectively.

Normalization of Diffuse Reflectance and Diffuse Transmittance Measurements. The transmittance, $T$, normalized by the signal obtained with the empty detector is considered to be absolute. The problem is more complex for diffuse reflectance. Only a collection system including an integrating sphere could give an exact indication about the absolute reflectance value $R$. A quantitative measurement hence implies the normalization of the spectra by using a reference. The choice of this reference is particularly important. The alkali halide salts are well-known for their excellent transmittance in the mid-infrared domain. A thick, totally opaque layer of $\mathrm{KBr}$ powder finely ground and carefully dehydrated should display in principle, in the absence of any absorbance, a diffuse reflectance equal to unity. For thinner, partially transparent layers, the conservation of energy implies $R+T$ $=1$.

In Table 1, the quantitative results obtained for two thin layers of a $\mathrm{KBr}$ powder with a particle diameter of less than $20 \mu \mathrm{m}$ are presented. The values are all taken at $\bar{\nu}=2247$ $\mathrm{cm}^{-1}$, the wavenumber corresponding to the $-\mathrm{C} \equiv \mathrm{N}$ chromophore absorption maximum. The high scattering power of $\mathrm{KBr}$ implies a total opacity for a thickness of the layer larger than $2 \mathrm{~mm}$. The reflectance of a 3-mm-thick layer can therefore be used for the normalization of the measurements. The sum of diffuse reflectance and transmittance values reported in Table 1, taking into account the uncertainties, tends to unity, indicating that absolute diffuse reflectance and diffuse transmittance are effectively measured.

Higher Sensitivity of Diffuse Transmittance Channel. In Figure 1, we show as an example two raw spectra obtained by diffuse reflectance FT-IR measurements of both Cab-OSil silica powders, treated up to surface saturation by the aminosilanes DMB and DMP.CN, respectively. The narrow and well-defined peak centered at $\bar{\nu}=2247 \mathrm{~cm}^{-1}$ is easily distinguishable and is characteristic of the nitrile function of the DMP.CN substituent.

A layer thickness of $1.3 \mathrm{~mm}$ is not sufficient to ensure the complete opacity of a Cab-O-Sil sample. The light intensity transmitted in a diffuse way can in consequence be measured by the photopyroelectric detector. ${ }^{21,22}$ The measured signals are normalized by the spectrum of the soot layer covering the PVDF film placed in the empty container yielding the absolute transmittance of the scattering layer.

Mixtures in different proportions of Cab-O-Sil powders, treated respectively by DMB and DMP.CN, are prepared. The absolute diffuse reflectance and transmittance are measured for different concentrations of the cyano group at $\bar{\nu}=2247 \mathrm{~cm}^{-1}$ and corrected for light absorption by the oxide substrate, as well as for the Fresnel reflection. ${ }^{22}$ The general

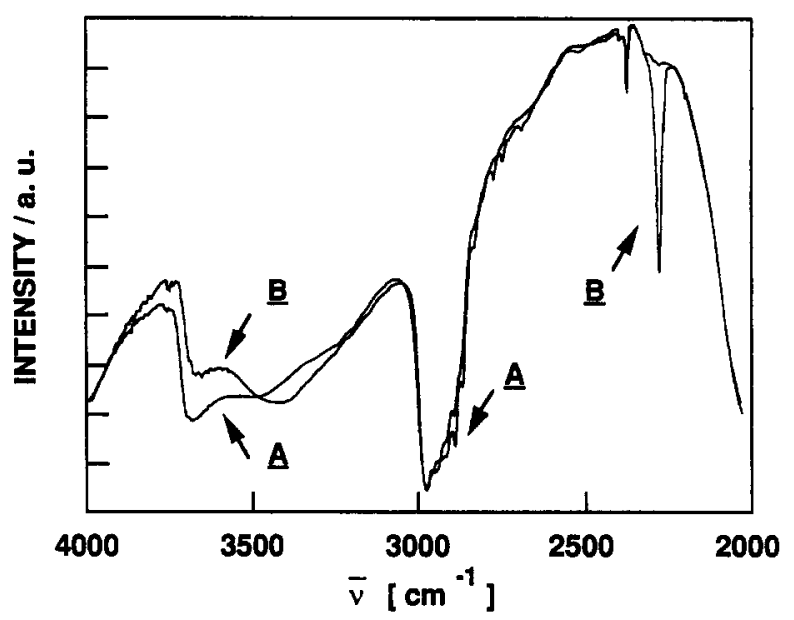

Flgure 1. Raw FT-IR diffuse reflectance spectra of a 1.3-mm-thick layer sample of Cab-O-Sil powder, treated up to complete surface coverage by (A) DMB and (B) DMP.CN aminosilane reagents. The spectra are typically obtained at a scan speed of $0.5 \mathrm{~cm} / \mathrm{s}$, with a resolution of $4 \mathrm{~cm}^{-1}$, and by accumulation of 250 scans.

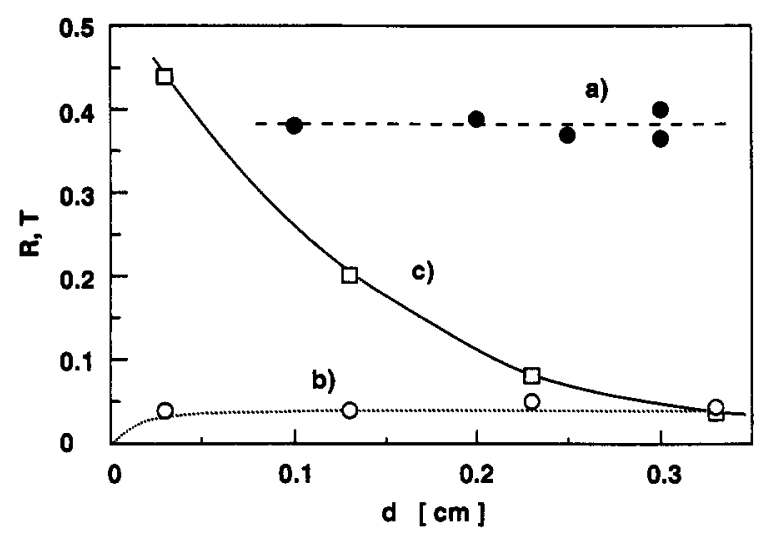

Figure 2. Effect of the depth of the layer $d$ on the absolute diffuse reflectance and transmittance of derlvatized LIChrosorb and Cab-O-SII sllica powder samples. Reported values are measured at the maximum of the absorption peak of the $\mathrm{CN}$ group at $\bar{\nu}=2247 \mathrm{~cm}^{-1}$. (a) Diffuse reflectance of various LIChrosorb sillca layers treated up to surface saturation by DMB substtuents. (b) Diffuse reflectance of various layers of Cab-O-Sil powder treated by DMP.CN aminosilane. (c) Diffuse transmittance of Cab-O-Sil powder under the same conditions as (b).

hyperbolic solutions of the Kubelka-Munk equation allow the considerations of diffuse reflectance and diffuse transmittance of the sample simultaneously. These solutions are adjusted for both sets of experimental data using a modified Marquardt nonlinear least-squares fitting procedure. ${ }^{31,32} \mathrm{Re}-$ sults are reported in ref 22 . An excellent correlation between the respective transmittance and reflectance measurements is obtained, and the scattering coefficient value at $\bar{\nu}=2247$ $\mathrm{cm}^{-1}$ is determined. In such a case, the absorption coefficient $K$ as well as the scattering coefficient $S$ can be determined simultaneously. Moreover, it was observed that the transmittance channel is more sensitive to concentration variations, therefore this channel can be used advantageously for analysis.

In Figure 2a, we report the reflectance of a LiChrosorb powder derivatized by DMB as a function of the depth of the scattering layer. Results show that the reflectance at the absorption maximum of the $\mathrm{CN}$ group does not vary between 1 and $3 \mathrm{~mm}$. No transmittance can be measured for layers

(31) Marquardt, D. W. Chem. Eng. Prog. 1959, 55, 65.

(32) Marquardt, D. W. J. Soc. Ind. Appl. Math. 1963, 11, 431. 
thicker than $1 \mathrm{~mm}$ which have therefore to be considered as pseudoinfinite with $R=R_{\infty}=0.38 \pm 0.02$.

For chemically treated Cab-O-Sil powder (Figure $2 \mathrm{~b}$ ), the measured reflectance $R=0.044 \pm 0.002$ as a function of the depth of the layer between 0.3 and $3.3 \mathrm{~mm}$ is much lower than the measured reflectance for LiChrosorb. Even though the scattering power of Cab-O-Sil is smaller than the one characterizing LiChrosorb powder, the reflectance value seems not to change beyond $300 \mu \mathrm{m}$ depth. Figure $2 \mathrm{c}$ shows that the phenomenon is completely different for the transmittance measurements. There is a very important decrease of transmittance from $T=0.44$ at $0.3 \mathrm{~mm}$ to about one-tenth of this value at $3.3 \mathrm{~mm}$. As the reflectance is not affected, such a variation is due to the absorption of the treated silica. This observation shows the higher sensitivity of the transmittance measurements to the absorption of the sample.

A similar conclusion has been drawn from the computer model simulations for the case of a new discontinuous multilayer theory. ${ }^{21}$ The transmittance in that case is more sensitive than the reflectance to the number of the particle layers. These observations indicate the necessity of a reconsideration of the total opacity criterion generally used in the literature. Indeed, the fact that there exists a depth beyond which the reflectance no longer increases does not necessary imply the absence of any transmitted light through thicker layers.

Effect of Application of Pressure on Scattering Coefficient of Powder. The application of a mechanical pressure on the surface of the powder can not ascertain a homogeneous density in the depth of the layer. This method is usually employed to level the powder surface and to obtain reproducible surfaces for reflectance measurements. In a recent paper, ${ }^{22}$ we have shown that the variation of the scattering coefficient with the depth of the layer can dramatically influence the reflectance value of the layer without necessarily affecting the mean value of this coefficient. In order to separate the effects of applied pressure from those of the apparition of inhomogeneities of the optical properties, such as absorption and scattering coefficients, samples constituted of mixtures of Cab-O-Sil powder with different concentrations of the $\mathrm{CN}$ group on their surfaces have been prepared in a particular way: An amount of the powder corresponding to about one tenth of the sample holder volume is compressed using a cylindrical piston penetrating inside the sample holder. A known pressure is applied by a dynamometric press and maintained for $1 \mathrm{~min}$. A same amount of the powder is poured on the top of this first layer and compressed in the same way. Thus, the first sublayer is submitted for a second time to a mechanical pressure. Nevertheless, as the maximum compaction is achieved already at the first compression, the density does not change anymore. This operation is repeated until a $3-\mathrm{mm}$ depth sample constituted of 10 superimposed sublayers is made. Each sublayer is thin enough to consider that absorption and scattering coefficients $K$ and $S$ are effectively constant within the depth of the whole sample.

Three different series of reflectance measurements have been done this way by application of increasing pressures of $5.89 \times 10^{6}, 1.18 \times 10^{7}$, and $1.05 \times 10^{8} \mathrm{~Pa}$. In each case, the density has been determined by weighing the sample holder. The results are presented in Figure 3 and Table 2. The absolute

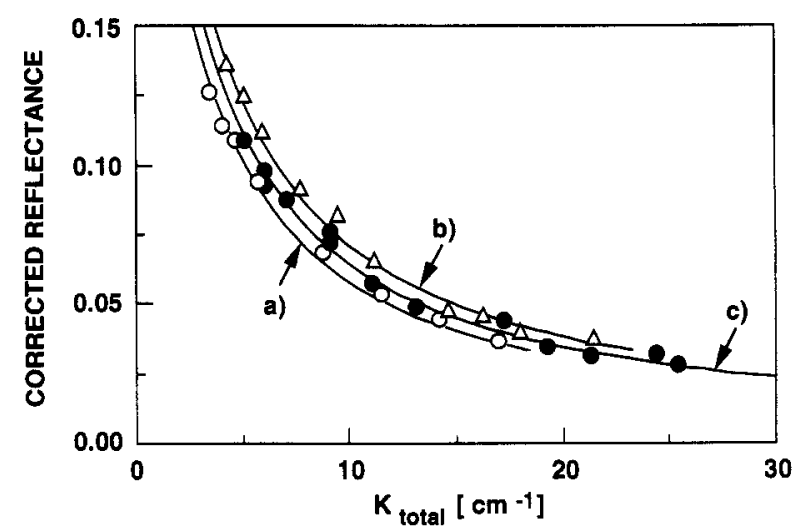

Figure 3. Absolute diffuse reflectance of mixtures in various proportions of both Cab-O-Sil powders treated up to surface coverage by DMB and DMP.CN aminosilanes, respectively. The samples are stacks of 10 thin layers, each of which was compressed under a pressure of (a) 58.9 $\times 10^{5}$, (b) $117.7 \times 10^{5}$, and (c) $1054.5 \times 10^{5} \mathrm{~Pa}$. Solld curves display the best fit of parameters in Kubelka's equation to the experimental results.

\begin{tabular}{|c|c|c|}
\hline pressure $\left(10^{5} \mathrm{~Pa}\right)$ & $\rho(\mathrm{g} / \mathrm{L})$ & $S\left(\mathrm{~cm}^{-1}\right)$ \\
\hline $\begin{array}{c}\sim 0 \\
58.9 \\
117.7 \\
1054.5\end{array}$ & $\begin{array}{l}130 \\
170 \\
220 \\
260\end{array}$ & $\begin{array}{l}1.25 \\
1.30 \\
1.65 \\
1.47\end{array}$ \\
\hline
\end{tabular}

a The mean value in the absence of an applied pressure has been established for the experimental results reported in ref 22 , where profiles of scattering and absorbtion coefficients were taken into account.

reflectance corrected for the Fresnel reflection ${ }^{22}$ increases with increasing pressure up to $1.18 \times 10^{7} \mathrm{~Pa}$ and decreases by a further increase of pressure up to $1.05 \times 10^{8} \mathrm{~Pa}$. For each family of the results, the hyperbolic model of Kubelka ${ }^{15}$ is used with success, and one can extract the scattering coefficient $S$ without introduction of any absorption or scattering coefficient profile in the depth of the layer. ${ }^{22}$

For the smallest pressure, there is an increase of the compaction density of $40 \%$. By application of further pressures, one obtains a density up to two times higher than the 'natural' density of the powder. The changes in the scattering coefficient follow the variations in the reflectance values. For Cab-O-Sil which is a fluffy powder, this is related to an increase of the number of the scattering centers by unit of volume in the first compaction phase. In a second phase, the number of the interfaces and thus the scattering coefficient decreases.

Griffiths et al..$^{7}$ reported an important decrease of the scattering coefficient following application of a pressure on their model systems constituted of dispersed caffeine in potassium chloride. The $\mathrm{KCl}$ powder, as well as $\mathrm{KBr}$ employed usually as a dilutant, is a compact powder formed by angular particles. A pressure of $6 \times 10^{7}-10^{9} \mathrm{~Pa}$ can just rearrange the position of the particles, which will tend to become aligned. Here, in the second phase of the compression of Cab-O-Sil powder, a similar behavior is hence observed.

A similar series of experiments has been carried out with the samples of LiChrosorb (Figure 4). Results are reported in Table 3. For LiChrosorb powder, which has a higher density and a porous morphology, the scattering coefficient increases 


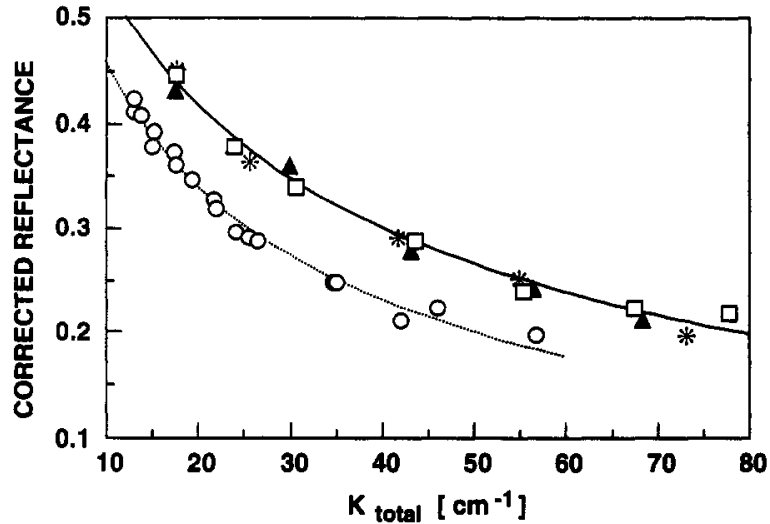

Figure 4. Absolute diffuse reflectance of mixtures in different proportions of both the LiChrosorb sillica powders treated up to surface coverage by DMB and DMP.CN aminosilanes, respectively. 3-mmthick samples are formed by stacking 10 thin layers, each individually compressed under a pressure of $58.9 \times 10^{5}(-\Delta-), 117.7 \times 10^{5}$ $(-*-)$, and $1054.5 \times 10^{5} \mathrm{~Pa}(-\square-)$. For comparison, values obtained for a noncompressed powder (.......) are also reported. Solld curves represent the best fit of parameters in Kubelka's equation to the experimental results.

Table 3. Results of Fitting of Scattering Coofflelent $S$ in Kubelka's Model to Expertmental Data of Flgure $4^{*}$

$\begin{array}{clc}\text { pressure }\left(10^{5} \mathrm{~Pa}\right) & \rho(\mathrm{g} / \mathrm{L}) & S\left(\mathrm{~cm}^{-1}\right) \\ \sim 0 & 350-370 & 26.5 \\ 58.9 & 480 & 48.1 \\ 117.7 & 500 & 49.2 \\ 1054.5 & 500 & 47.0\end{array}$

a The mean value in the absence of any applied pressure is reported for comparison.

in the first phase and then remains essentially constant upon further application of pressure. The scattering coefficient $S$ attains a plateau value $48 \pm 1 \mathrm{~cm}^{-1}$. A maximum density of about $35 \%$ higher than the natural density of the powder is obtained already for the smallest pressure, and hence the compaction does not change by further application of pressure. For a relatively low compression compared to that of CabO-Sil, a relatively large increase of about two times of the scattering coefficient is observed. Since the number of optical interfaces inside porous LiChrosorb does not increase by application of a pressure, the coefficient $S$ should not vary. Electron microscopy studies show that the LiChrosorb powder grains might break up under pressure, so one has to be careful in interpreting the scattering coefficient data in this case.

Influence of Chemical Environment of the Functional Group on Its Molar Extinction Coefficient. In order to study the influence of chemical environment of the $\mathrm{CN}$ chromophore on its absorption coefficient, two series of the experiment have been carried out. In the first series, two samples of LiChrosorb have been treated up to a maximum surface coverage with DMB and DMP.CN separately. Mixtures with different proportions of the two powders are prepared in order to vary the concentration of the cyano group. In the second series, a sample of LiChrosorb powder is reacted with a mixture of the two aminosilanes. The variation of the concentration of cyano on the same surface is obtained by mixing different proportions of the two aminosilanes before the reaction. In this case, the environment of the cyano group is expected to

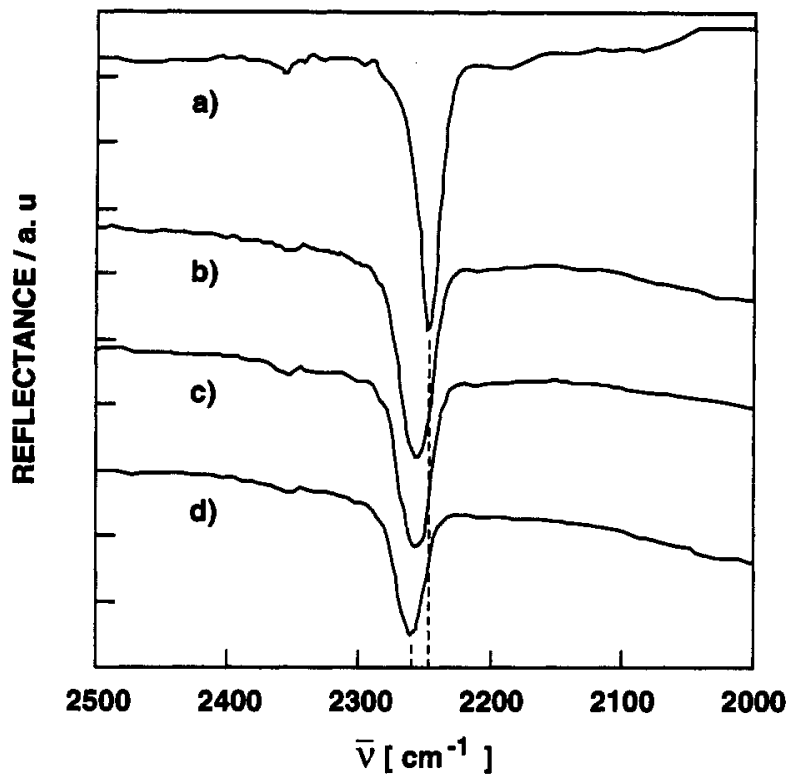

Flgure 5. Diffuse reflectance spectra measured for a LiChrosorb sillice powder treated by the aminosilane DMP.CN resulting in varlous fractions of surface coverage: (a) 1.0, (b) 0.71 , (c) 0.51 , and (d) 0.25 . The maximum of the absorption peak is shifted toward the blue as the surface coverage increases. The position of base lines is arbltrary.

depend on the relative rate of coverage of silica by the DMP.CN substituent and on the nature of the other molecules adsorbed on the same surface.

Figure 5 shows four different diffuse reflectance spectra of the LiChrosorb powder, which is partially covered with various concentration of DMP.CN substituents. $\mathrm{C}, \mathrm{H}$, and $\mathrm{N}$ elementary analyses allow determination in each case of the surface coverage by the siloxy groups. The large IR absorption of hydroxyl groups on the silica surface results in a strongly varying baseline. Quantitative diffuse reflectance analysis of the cyano group is thus impossible in this case. The maximum absorption peak of the cyano group for a total surface coverage is positioned at $2247 \mathrm{~cm}^{-1}$. For the smaller fractions of DMP.CN, one can observe a logical decrease of the band intensity together with a blue shift of the peak. At a quarter of the surface covered with DMP.CN substituents, the maximum of the absorption peak is shifted by $13 \mathrm{~cm}^{-1}$ as compared to the completely covered surface. This shift to higher energies may be attributed to the formation of hydrogen bonds $\mathrm{CN}$... OH between the cyano and hydroxyl groups in their environment. Such an effect has been reported for the aliphatic nitrile groups in a protic solvent. ${ }^{33}$ Once the silylation reaction is completed with the two aminosilanes simultaneously and saturation of the silica surface is attained, the hydroxyl groups are mostly eliminated, and the above-mentioned effect is no longer expected. The variation of the molar fraction of the DMP.CN substitutents balanced by that of the DMB substitutents does not give rise to any shift of the absorption maximum of the nitrile group, which remains at $\bar{\nu}=2247$ $\mathrm{cm}^{-1}$.

Figure 6 shows the absolute diffuse reflectance at $\bar{\nu}=2247$ $\mathrm{cm}^{-1}$ for the LiChrosorb powder submitted to a reaction with a mixture of both aminosilanes DMB and DMP.CN. The phenomenological absorption coefficient is calculated knowing

(33) Williams, I. Ind. Eng. Chem. 1923, 15, 154. 


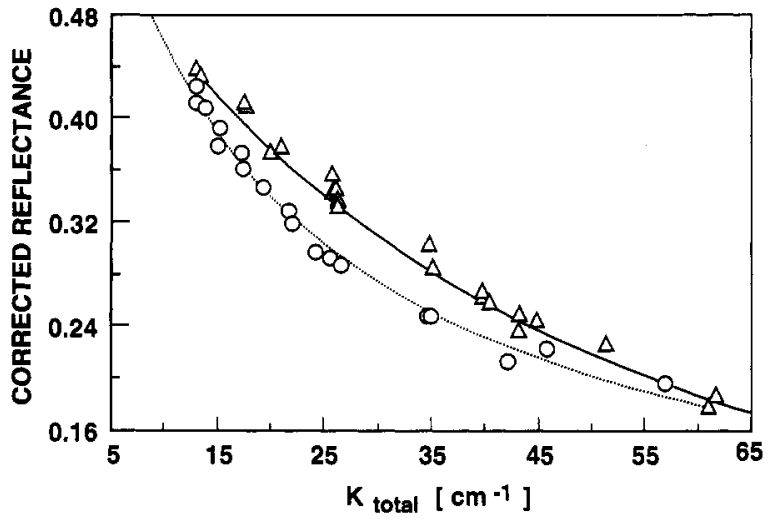

Flgure 6. Diffuse reflectance at $\bar{\nu}=2247 \mathrm{~cm}^{-1}$ of a LiChrosorb silica powder, treated by a mixture of both aminosilanes DMB and DMP.CN $(-\Delta-)$. For comparison, data obtained for mixtures in different proportions of powders treated separately by both aminosillanes DMB and DMP.CN up to surface saturation are also reported (... O...).

the molar fraction of DMP.CN and the molar extinction coefficient $\epsilon \cong 27 \mathrm{~L} \mathrm{~mol}^{-1} \mathrm{~cm}^{-1}$ determined previously for the $\mathrm{CN}$ chromophore. ${ }^{22}$ The optical absorption of the silica powder itself has also been taken into account. A quantitative model derived from the hyperbolic solutions of Kubelka for the differential equations of Kubelka-Munk developed in a recent paper is applied to the experimental results. The best Marquardt least-squares adjustment of the results gives a scattering coefficient of $S=39.7 \mathrm{~cm}^{-1}$, which is larger than $S=26.2 \mathrm{~cm}^{-1}$ obtained for mixtures of two powders treated up to saturation of their surfaces by both aminosilanes respectively. A first hypothesis put forward to explain this difference is a change in the morphology of the powders used in both cases. No difference is observed in the density of the two powders, nor is any change in the morphology of the grains noticed under the electron microscope. The two groups of results reported in Figure 6 and the two theoretical fits show a good coincidence of the two extreme points $y(B)=0$ and $y(B)=1$ where the samples must effectively be nondiscernible. This observation suggests identical scattering coefficients for both sets of samples equal to $S=26.2 \mathrm{~cm}^{-1}$. In these conditions, only an error on the evaluation of the $K_{\text {tot }}$ coefficients can explain the difference observed between the scattering coefficients in the two sets of experiments. The molar extinction coefficient of the chromophore can indeed be a function of its molar fraction on the silica mixed treated surface. If this is the case, $K_{\text {tot }}$, which has been calculated using $\epsilon=27 \mathrm{~L} \mathrm{~mol}^{-1} \mathrm{~cm}^{-1}$, is not correct for the various concentrations, and the difference observed between the two curves is due to the error in the determination of $K_{\text {tot }}$.

The two curves in Figure 6 can be superimposed by identifying at equal reflectance the absorption coefficient to that calculated for the mixture of the powders. Once the real absorption coefficients $K$ are determined, one can obtain the molar extinction coefficient corresponding to each fraction $y(B)$ by using the linear relation

$$
K=2 \ln (10) \epsilon \rho c_{\mathrm{m}}
$$

where $\epsilon$ is the chromophore molar extinction coefficient (L $\left.\mathrm{mol}^{-1} \mathrm{~cm}^{-1}\right), \rho$ is the solid density $\left(\mathrm{g} \mathrm{L}^{-1}\right)$, and $c_{\mathrm{m}}$ is the mass

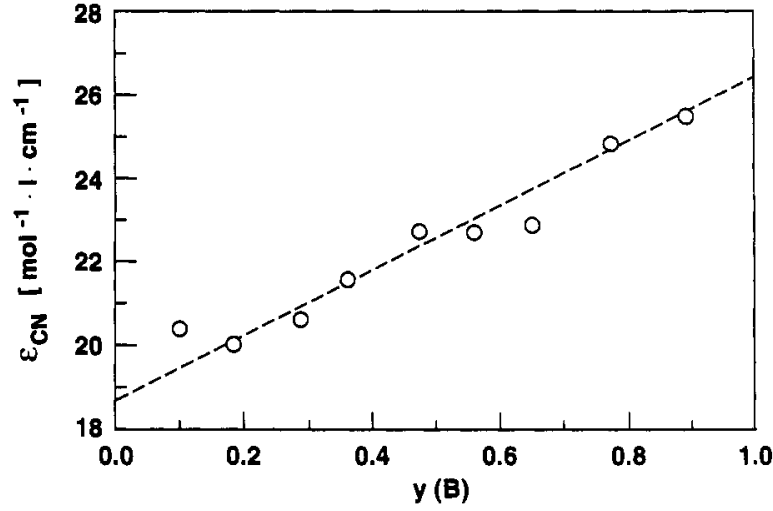

Flgure 7. Molar extinction coefficient of the cyano functional group anchored on a LiChrosorb powder treated by a mixture of both aminosilanes DMB and DMP.CN as a function of the varlous molar fractions $Y(B)$ of substituents carrying a $C N$ function.

concentration of the substituent carrying the $\mathrm{CN}$ group (mol $\left.\mathrm{g}^{-1}\right)$ :

$$
c_{\mathrm{m}}(B)=\frac{y(B) \Gamma_{\mathrm{tot}}}{\frac{1}{S_{\mathrm{A}}}+\Gamma_{\mathrm{tot}}\left[y(B) M_{(\mathrm{B})}+[1-y(B)] M_{(\mathrm{A})}-\delta\right]}
$$

In the latter expression, $y(B)$ represents the molar fraction of the substituent containing the $\mathrm{CN}$ group, $M(A)$ and $M(B)$ are the molecular weight of the siloxy radical of DMB and DMP.CN, respectively $\left(\mathrm{g} \mathrm{mol}^{-1}\right), \Gamma_{\text {tot }}$ is the total surface concentration of the substitutents $\left(\mathrm{mol} \mathrm{m}^{-2}\right), S_{\mathrm{A}}$ is the specific area of the surface of silica before reaction $\left(\mathrm{m}^{2} \mathrm{~g}^{-1}\right)$, and $\delta$ is a correction factor taking into account the desorbed water and the substitution of one proton during the silylation reaction $(\mathrm{g} \mathrm{mol}-1){ }^{20}$

The results of this operation are presented in Figure 7. For a complete surface coverage of silica by DMP.CN substituents, an extinction coefficient of $\epsilon=27 \mathrm{~L} \mathrm{~mol}^{-1} \mathrm{~cm}^{-1}$ is found. This value tends in a linear way to $\epsilon=20 \mathrm{~L} \mathrm{~mol}^{-1}$ $\mathrm{cm}^{-1}$, as the molar fraction of the chromophore tends to zero. This phenomena is the result of a change of the environment of the cyano group. For $y(B)=1$, this implies an important interaction between vicinate nitrile groups. An inductive effect of the nitrogen electronegative group on the electrodefficient carbon of the neighbor group tends to improve the polarization of the triple bond under its mesomer form $-\mathrm{C}^{+}=\mathrm{N}^{-}$, which is characterized by a higher absorption. Diluted in the nonpolar DMB substitutent, the chromophore extinction coefficient approaches that of capronitrile in solution in $n$-dodecane $\left(\epsilon=20 \mathrm{~L} \mathrm{~mol}^{-1} \mathrm{~cm}^{-1}\right)$.

\section{ACKNOWLEDGMENT}

The authors would like to thank Dr. Roland Cloux for the preparation of the aminosilane reagents and Prof. Andreas Mandelis for fruitful discussions. Financial support of this work has been provided by the Fonds National Suisse de la Recherche Scientifique.

Recelved for review January 3, 1994. Accepted April 20, 1994.

- Abstract published in Advance ACS Abstracts, May 15, 1994. 